\title{
THE LEAST NUMBER OF COINCIDENCE POINTS ON SURFACES
}

\author{
JERZY JEZIERSKI \\ (Received 17 January 1991; revised 22 April 1992) \\ Communicated by J. H. Rubinstein
}

\begin{abstract}
Bo Ju Jiang introduced an invariant lying in the braid group which is the best lower bound of the number of fixed points in a homotopy class of a given pair of self maps of a surface. Here we modify this construction to get a lower bound of the number of coincidence points of a pair of maps between two closed surfaces.
\end{abstract}

1991 Mathematics subject classification (Amer. Math. Soc.): primary 55M20, secondary 57R99.

\section{Introduction}

The long standing problem, whether or not the Nielsen number $([2,8])$ is the best lower estimate of the number of fixed points of self-maps of surfaces, was answered negatively by Bo Ju Jiang [9]. In [10], for any self-map of a surface an element in the braid group is defined and its 'width' turns out to be the best lower bound of fixed points.

In this paper we introduce a similar element for a pair of maps $f, g: M \rightarrow N$ between closed surfaces and we prove that its width is the best lower bound of coincidence points. Then in Section 3 we show that after abelianization this element is sent onto the classical Nielsen invariant. The method of this section is simpler than those of [10, Section 5]: we avoid commutator and Fox calculus. Finally, since the above theory requires the assumption $\pi_{2} N=0$, we show that for pairs of maps into $S^{2}$ or $R P^{2}$ (the only surfaces with $\pi_{2} N \neq 0$ ) the classical Nielsen number can always be realized.

To get the minimalization theorem, we use homotopies deforming both maps. However it follows from [1] that the least number of coincidence points may always be obtained by a pair of homotopies in which one map is constant.

(C) 1995 Australian Mathematical Society 0263-6115/95 $\$ A 2.00+0.00$ 

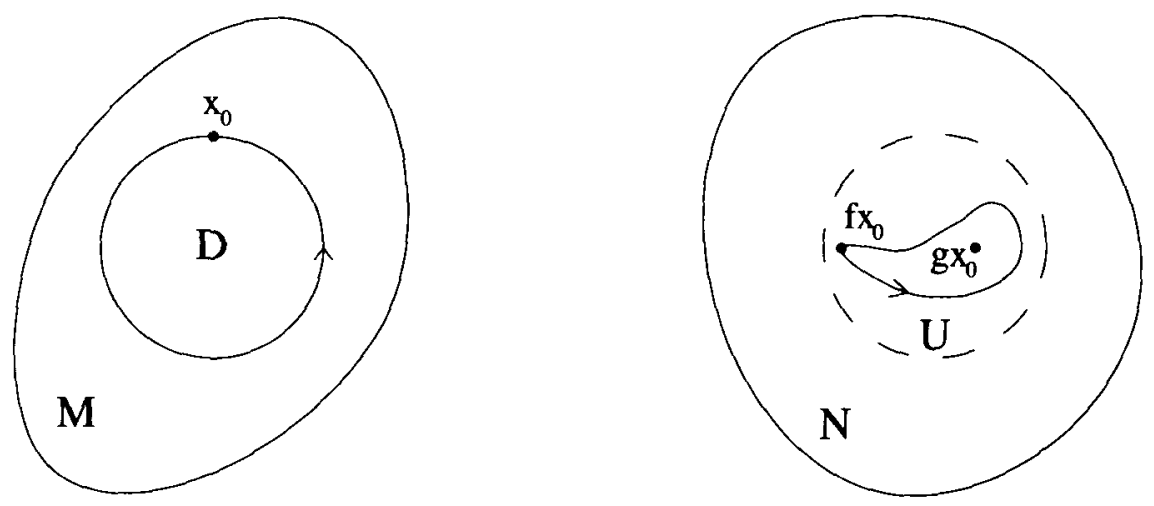

FIGURE 1

\section{An algebraic formulation of coincidence problem}

Let $M$ and $N$ be closed connected two-dimensional manifolds and let $\pi_{2} N=0$. We will consider a pair of continuous maps $f, g: M \rightarrow N$ and its coincidence set $\Phi(f, g)=\{x \in M: f x=g x\}$. We will look for the lower bound of the cardinality $\# \Phi(f, g)$ in the homotopy classes of $f, g$. Let us fix a point $x_{0} \notin \Phi(f, g)$. The following lemma shows that we need only consider homotopies rel $x_{0}$.

LEMMA 1.1. (Compare [9]) Let $f, g ; f^{\prime}, g^{\prime}: M \rightarrow N$ be homotopic pairs of maps such that $f x_{0}=f^{\prime} x_{0} \neq g x_{0}=g^{\prime} x_{0}$. Then the pair $f, g$ is homotopic rel $x_{0}$ to a pair $f^{\prime \prime}, g^{\prime \prime}$ such that $\Phi\left(f^{\prime \prime}, g^{\prime \prime}\right)=\Phi\left(f^{\prime}, g^{\prime}\right)$.

Proof. Let $F, G: M \times I \rightarrow N$ be a pair of homotopies between $f, g$ and $f^{\prime}, g^{\prime}$. Then the restrictions $F, G:\left\{x_{0}\right\} \times I \rightarrow N$ are homotopic rel $\left(\left\{x_{0}\right\} \times \delta I\right)$ to some maps $\alpha, \beta$ satisfying $\alpha\left(x_{0}, t\right) \neq \beta\left(x_{0}, t\right)$ for $t \in[0,1]$. The homotopy extension property of $\left(M \times I, M \times \delta I \cup\left\{x_{0}\right\} \times I\right)$ gives us homotopies $F^{\prime}, G^{\prime}$ from $f, g$ to $f^{\prime}, g^{\prime}$ with $F^{\prime}\left(x_{0}, t\right) \neq G^{\prime}\left(x_{0}, t\right)$. Then $F^{\prime}(x, t) \neq G^{\prime}(x, t)$ in some neighbourhood $W$ of $x_{0}$. Let $\lambda: M \rightarrow[0,1]$ be a Urysohn function satisfying $\lambda\left(x_{0}\right)=0$ and $\lambda(M-W)=1$. Then $F^{\prime \prime}(x, t)=F^{\prime}(x, \lambda(x) t), G^{\prime \prime}(x, t)=G^{\prime}(x, \lambda(x) t)$ are homotopies rel $x_{0}$ and we put $\left.f^{\prime \prime}=F^{\prime \prime}(., 1), g^{\prime \prime}=G^{\prime \prime}(., 1)\right)$.

Now we fix a closed ball $D \subset M$ with $x_{0}$ on its boundary and an open ball $U \subset N$ containing $f x_{0}$ and $g x_{0}$ (Figure 1). Let us also fix orientations of $D$ and $U$. Let $b$ be a loop in $U-g x_{0}$ based at $f x_{0}$ running once around $g x_{0}$ in positive sense of $U$. 

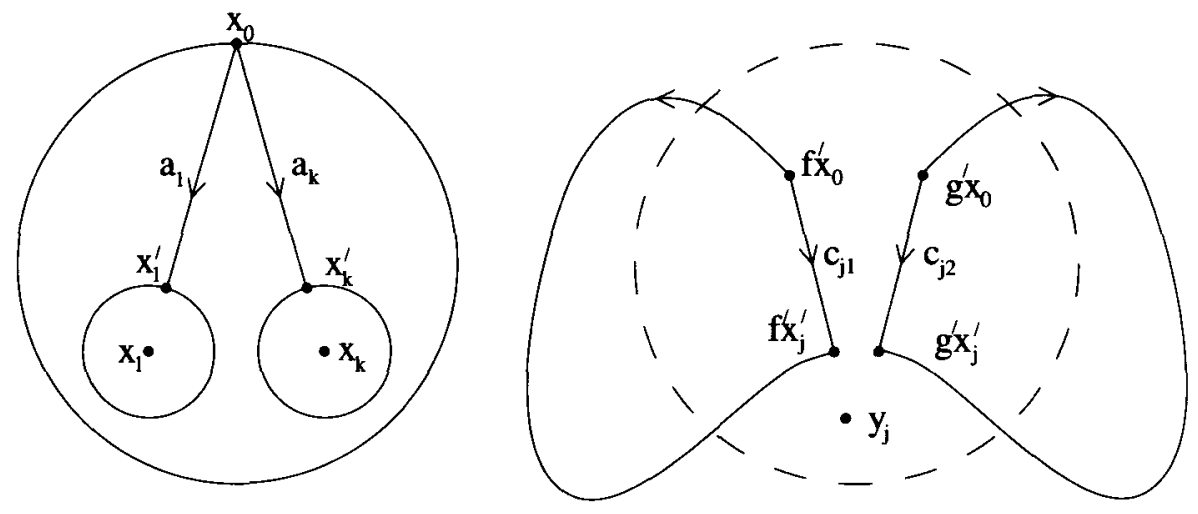

FIGURE 2

LEMMA 1.2. Let $f, g: M \rightarrow N$ be as above. Let $k \geq 0, i_{1}, \ldots, i_{k} \in Z$. Then the following two conditions are equivalent.

(I) The pair $f, g$ is homotopic rel $x_{0}$ to a pair $f^{\prime}, g^{\prime}$ with exactly $k$ coincidence points of indices $i_{1}, \ldots, i_{k}, \Phi\left(f^{\prime}, g^{\prime}\right) \subset$ int $D, f\left(\Phi\left(f^{\prime}, g^{\prime}\right)\right) \subset U$ (the indices are taken with respect to fuxed orientations of $D$ and $U$ ).

(II) There exist a homomorphism $\Phi: \pi_{1}\left(M-\operatorname{int} D, x_{0}\right) \rightarrow \pi_{1}(N \times N-$ $\left.\Delta,\left(f x_{0}, g x_{0}\right)\right)$ and elements $v_{1}, \ldots, v_{k} \in \pi_{1}\left(N \times N-\Delta,\left(f x_{0}, g x_{0}\right)\right)$ such that the diagram

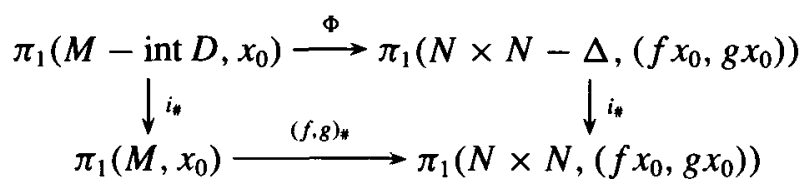

commutes and $\Phi(T)=v_{1} B^{i_{1}} v_{1}^{-1} \ldots v_{k} B^{i_{k}} v_{k}^{-1}$ (where $T$ denotes the homotopy class of bd $D$ ).

PROOF. (I) implies (II). We assume that $f, g$ are homotopic rel $x_{0}$ to a pair $f^{\prime}, g^{\prime}$ that $\Phi\left(f^{\prime}, g^{\prime}\right)=\left\{x_{1}, \ldots, x_{k}\right\} \subset$ int $D$ and $y_{i}=f^{\prime} x_{i}=g^{\prime} x_{i} \in U$. Let us fix closed disjoint balls $D_{i} \subset D$ such that $x_{i} \in$ int $D_{i}, f^{\prime} D_{i} \cup g^{\prime} D_{i} \subset U$, a point $x_{i}^{\prime} \in$ bd $D_{i}$ and an arc $a_{i}$ joining in $D-\cup D_{j}$ the points $x_{0}$ and $x_{i}^{\prime}$. Let $T_{i}$ be a loop of $\delta D_{i}$ based at $x_{i}^{\prime}$ oriented in a positive sense, $(i=1, \ldots, k)$.

We define $\Phi=\left(f^{\prime}, g^{\prime}\right)_{\#}: \pi_{1}\left(M-\right.$ int $\left.D, x_{0}\right) \rightarrow \pi_{1}\left(N \times N-\Delta,\left(f x_{0}, g x_{0}\right)\right)$. Then the diagram commutes. For $j=1, \ldots, k$ we fix paths $c_{j 1}, c_{j 2}$ joining $f^{\prime} x_{0}$ with $f^{\prime} x_{j}^{\prime}$ and $g^{\prime} x_{0}$ with $g^{\prime} x_{j}^{\prime}$ in $U$ respectively satisfying $c_{j 1}(t) \neq c_{j 2}(t)$. Then

$$
\Phi(T)=\left(f^{\prime}, g^{\prime}\right)_{\#} T=\left(f^{\prime}, g^{\prime}\right)_{\#}\left(a_{1} T_{1} a_{1}^{-1} \ldots a_{k} T_{k} a_{k}^{-1}\right), \quad \text { and }
$$




$$
\left(f^{\prime}, g^{\prime}\right)_{\#}\left(a_{j} T_{j} a_{j}\right)=\left(v_{j 1} c_{j 1}\left(f^{\prime} T_{j}\right) c_{j 1}^{-1} v_{j 1}^{-1}, v_{j 2} c_{j 2}\left(g^{\prime} T_{j}\right) c_{j 2}^{-1} v_{j 2}^{-1}\right)
$$

where $v_{j}=\left(v_{j 1}, v_{j 2}\right)=\left(\left(f^{\prime} a_{j}\right) c_{j 1}^{-1},\left(g^{\prime} a_{j}\right) c_{j 2}^{-1}\right) \in \pi_{1}\left(N \times N-\Delta, f x_{0}, g x_{0}\right)$.

On the other hand

$$
\left\langle c_{j 1}\left(f^{\prime} T_{j}\right) c_{j 1}^{-1}, c_{j 2}\left(g^{\prime} T_{j}\right) c_{j 2}^{-1}\right\rangle \in \pi_{1}(U \times U-\Delta)=\pi_{1}\left(R^{2} \times R^{2}-\Delta\right)=Z
$$

and the above group is generated by $B$. Thus we get

$$
\left.\left(f^{\prime}, g^{\prime}\right)_{\#}\left(a_{j} T_{j} a_{j}^{-1}\right)=v_{j} B^{i_{j}} v_{j}^{-1} \quad \text { (with } i_{j}=\text { ind }\left(f^{\prime}, g^{\prime}: x_{j}\right)\right)
$$

which implies

$$
\Phi(T)=v_{1} B^{i_{1}} v_{1}^{-1} \ldots v_{k} B^{i_{k}} v_{k}^{-1} .
$$

(II) implies (I). Let us fix as above: balls $D, D_{j} \subset M, U \subset N$, points $x_{j} \in$ int $D_{j}$, $x^{\prime} \in$ bd $D_{j}, y_{j} \in U$ and paths $a_{j}, c_{j 1}, c_{j 2}$. Let us define the maps $f^{\prime}, g^{\prime}$ on the arc $a_{j}$ by $f^{\prime} a_{j}=v_{j 1} c_{j 1}, g^{\prime} a_{j}=v_{j 2} c_{j 2}$ and then on $D_{j}$ to get the unique coincidence point $x_{j}$ of index $i_{j}$. We extend these maps by a retraction onto the whole of $D$. Let us notice that then

$$
\left(f^{\prime}, g^{\prime}\right)_{\#} T=v_{1} B^{i_{1}} v_{1}^{-1} \ldots v_{k} B^{i_{k}} v_{k}^{-1} \in \pi_{1}(N \times N-\Delta) .
$$

Now we extend $f^{\prime}, g^{\prime}$ onto $M$ so that $M$-int $D$ is sent into $N \times N-\Delta$. At first we extend $\left(f^{\prime}, g^{\prime}\right)$ on the 1-skeleton $M^{(1)} \subset M$-int $D$ to get a commutative diagram

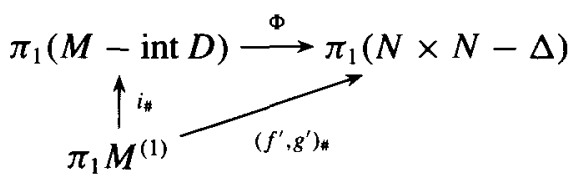

and this may be extended onto each 2-simplex in $M$-int $D$. Pasting the last map with the part already defined on $D$ we obtain the desired maps $f^{\prime}, g^{\prime}: M \rightarrow N$. It remains only to show that $f^{\prime}, g^{\prime}$ are homotopic to $f, g$. But the vertical homomorphisms in the commutative diagram

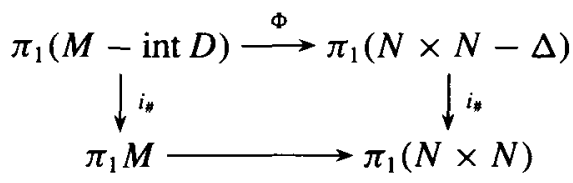

and the ones in (II) are surjective. This implies $(f, g)_{\#}=\left(f^{\prime}, g^{\prime}\right)_{\#}$; hence, since $\pi_{1}(N \times N)=0$ the pairs $f, g$ and $f^{\prime}, g^{\prime}$ are homotopic. 


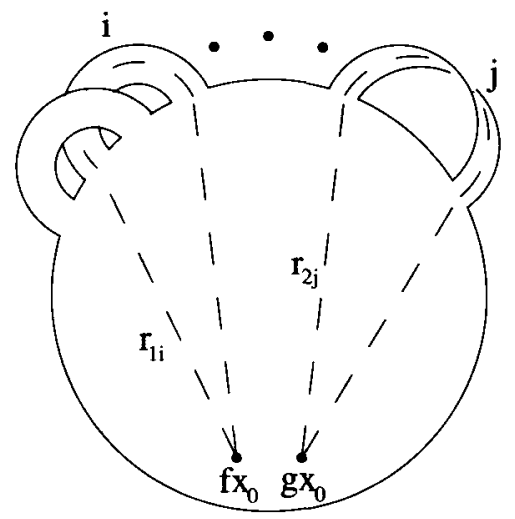

FigURE 3

\section{Braid groups}

We will use the presentation of the (pure 2-) braid group given in [10, Section 2]. We denote

$$
\begin{aligned}
& G=\pi_{1}\left(N \times N-\Delta,\left(f x_{0}, g x_{0}\right)\right) \\
& H=\operatorname{ker}\left(p_{1 \#}: \pi_{1}\left(N \times N-\Delta,\left(f x_{0}, g x_{0}\right)\right) \rightarrow \pi_{1}\left(N, f x_{0}\right)\right) \\
& K=\operatorname{ker}\left(i_{\#}: \pi_{1}\left(N \times N-\Delta,\left(f x_{0}, g x_{0}\right)\right) \rightarrow \pi_{1}\left(N \times N,\left(f x_{0}, g x_{0}\right)\right)\right) .
\end{aligned}
$$

If we remove a disk from the surface $N$ we obtain a two-dimensional disk with $r$ pairs of linked bands and $s$ twisted bands as shown on Figure 3.

We denote by $r_{1 j}, r_{2 j}$ the loops in $N$ based at $f x_{0}$ and $g x_{0}$ respectively as shown in Figure $3, j=1, \ldots, 2 r+s$. The $i_{k}$ image of $r_{k j}$ in $G$ will be denoted by $\rho_{k j}, k=1,2$. The group $G$ is generated by $\rho_{1 j}, \rho_{2 j}, B$ with the relations $2-1, \ldots, 2-5$ of $[10$, Theorem 1] (where $g=r, h=s, n=0$ ). We recall only that

$$
B=\left[\rho_{21}, \rho_{22}^{-1}\right] \ldots\left[\rho_{2,2 r-1}, \rho_{2,2 r}^{-1}\right] z_{2 r+1}^{2} \ldots z_{2 r+s}^{2}
$$

and that the right side is denoted by $R\left(\rho_{21}, \ldots, \rho_{2 r+s}\right)$.

Similarly we denote by $r_{j}$ the generators of $\pi_{1}\left(M, x_{0}\right)$. Now [10, Section 2] implies the following statements:

The group $H$ is a free group with the basis $\left\{\rho_{2 i}: i=1, \ldots, 2 r+s\right\}$.

The group $K=\operatorname{ker} i_{*}=H \cap \operatorname{ker} p_{2 *}$ is the normal subgroup of $H$ generated by $B$.

The map $p_{2 *}$ induces an isomorphism from the quotient group $\pi=H / K$ onto $\pi_{1}\left(N, g x_{0}\right)$. 
Thus $\pi$ is generated by $\left\{\rho_{2 j}\right\} j=1, \ldots, 2 r+s$ with the one relation $R\left(\rho_{21}, \ldots, \rho_{2,2 r+s}\right)=1$. The group $K$ is free on the basis $\left\{s B s^{-1} ; s \in S\right\}$ where $S$ is a minimal Schreier transversal with respect to the projection $H \rightarrow \pi$. We will also write $B^{k v}=v B^{k} v^{-1}$ and $B^{u+v}=B^{u} B^{v}$ for $u, v, B \in \Gamma$ (a group) and $k \in Z$. (This convention is not consistent with that of Jiang [10]).

We denote by $Z[\Gamma]$ the integral group ring and by $\hat{Z}[\Gamma]$ the set of integral combinations of elements from $\Gamma$ with non-commutative addition. Then $Z[\Gamma]$ is the abelianization of $\hat{Z}[\Gamma]$.

The 'exponential' homomorphism $\beta: \hat{Z}[G] \rightarrow K \beta(g)=B^{g}$ and a Schreier minimal transversal of $H \rightarrow \pi$ induce an isomorphism $\beta_{S}: \hat{Z}[\pi] \rightarrow K, \beta_{S}(g)=B^{\bar{g}}$ where $\bar{g}$ is a Schreier representative of $g$. Now let us return to our maps $f, g: M \rightarrow N$. They induce homomorphisms: $f_{\#}: \pi_{1}\left(M, x_{0}\right) \rightarrow \pi_{1}\left(N, f x_{0}\right), g_{\#}: \pi_{1}\left(M, x_{0}\right) \rightarrow$ $\pi_{1}\left(N, g x_{0}\right)$. Since $\left\{r_{j}\right\},\left\{r_{1 j}\right\},\left\{r_{2 j}\right\}$ are generators of the above groups, $f_{\sharp} r_{j}=f_{j}$, $g_{\#} r_{j}=g_{j}$ where $f_{j}, g_{j}$ are words in the letters $\left\{r_{1 j}\right\},\left\{r_{2 j}\right\}$ respectively.

Let us define $\sigma_{j}=f_{j}\left(\rho_{11}, \ldots, \rho_{1,2 r+s}\right) g_{j}\left(\rho_{21}, \ldots, \rho_{2,2 r+s}\right)$.

LEMMA 2.1. The condition (II) of Lemma 1.2 is equivalent to

(III) There exist elements $u_{j} \in K,(j=1, \ldots, 2 r+s)$ and $v_{j} \in G(j=1, \ldots, k)$ such that $R\left(u_{j} \sigma_{j}\right)=B^{i_{1} v_{1}+\ldots+i_{k} v_{k}}$ in $K$.

ProOF. (II) implies (III). By the diagram of (II), $\Phi\left(r_{j}\right)$ and $\sigma_{j}$ differ by an element of ker $j_{\#}=K$; hence $\Phi\left(r_{j}\right)=u_{j} \sigma_{j}$ for some $u_{j} \in K$.

Since $T=R\left(r_{j}\right)$,

$$
B^{i_{1} v_{1}+\ldots+i_{k} v_{k}}=\Phi(T)=\Phi\left(R\left(r_{j}\right)\right)=R\left(\Phi\left(r_{j}\right)\right)=R\left(u_{j} \sigma_{j}\right)
$$

(III) implies (II). The group $\pi_{1}\left(M\right.$-int $\left.D, x_{0}\right)$ has free basis $\left(r_{j}\right)$. We define $\Phi\left(r_{j}\right)=u_{j} \sigma_{j}$. Then $i_{\#} \Phi\left(r_{j}\right)=i_{\#}\left(u_{j} \sigma_{j}\right)=i_{\sharp} \sigma_{j}=(f, g)_{\#} i_{\#} r_{j}$ so the diagram in (II) commutes. Moreover

$$
B^{i_{1} v_{1}+\ldots+i_{k} v_{k}}=R\left(u_{j} \sigma_{j}\right)=R\left(\Phi\left(r_{j}\right)\right)=\Phi\left(R\left(r_{j}\right)\right)=\Phi(T)
$$

REMARK 2.2. If $N$ is orientable, the condition $v_{j} \in G$ in (II) and (III) may be replaced by $v_{j} \in H$ (see [10, (2.2)]) In the non-orientable case such reduction is also possible but then $i_{j}$ may change sign. In any case we may follow [10, Section 3$]$ to obtain

PROPOSITION 2.3. $M F(f, g)$ equals the minimal width of elements $R\left(u_{i} \sigma_{i}\right) \in K$ with respect to the basis $\left\{s B s^{-1}: s \in S\right\}$, where $S$ stands for a Schreier minimal transversal and $u_{1}, \ldots, u_{2 r+s}$ run over all $K$. 


\section{Connection with the Nielsen invariant}

In this section we will show that the elements appearing in Proposition 2.3 are sent by abelianization into the classical Nielsen invariant.

We identify the groups $\pi_{1}\left(N, f x_{0}\right), \pi_{1}\left(N, g x_{0}\right)$ by a path from $f x_{0}$ to $g x_{0}$ lying in $\bar{U}$. Then $r_{1 i}$ corresponds to $r_{2 i}$. We define the left action of $\pi_{1}\left(M, x_{0}\right)$ on $\pi=\pi_{1}\left(N, g x_{0}\right)$ by $r a=g_{\#}(r) a\left(f_{\#} r\right)^{-1}$. We denote by $\pi_{R}$ the orbit space of this action (the set of Reidemeister classes).

LEMMA 3.1. For any $x \in \Phi(f, g)$ and a path $r$ from $f x_{0}$ to $g x_{0}$ in $U$ the set

$$
\left\{\left((g u)\left(f u^{-1}\right) r\right\rangle: u \text { is a path in } M \text { from } x_{0} \text { to } x\right\}
$$

is exactly one orbit of $\pi_{R}$. Two points $x, y \in \Phi(f, g)$ are Nielsen equivalent if and only if they determine the same orbit.

PROOF. Notice that $\pi_{R}$ is equal to the set $\nabla\left(g, f: x_{0}, r^{-1}\right)$ as defined in [4]. Then apply [4, Lemma (1.2)].

COROLLARY 3.2. The above formula determines an injection $I: \Phi^{\prime}(f, g) \rightarrow \pi_{R}$ : for any $x \in \Phi(f, g)$ we put $I(x)=\left\langle(g u)(f u)^{-1} r\right\rangle_{R}$ where $u$ is a path from $x_{0}$ to $x$ and $\Phi^{\prime}$ denotes the set of Nielsen classes.

Definition 3.3. Let $M$ and $N$ be oriented. The element $\sum_{A}$ ind $(f, g: A) I(A) \in$ $Z\left[\pi_{R}\right]$ (where the summation runs over the set of Nielsen classes) will be called the Nielsen invariant of $f, g$. When either of $M$ or $N$ is not orientable then the element $\sum_{A}$ ind $_{2}(f, g: A) I(A) \in Z_{2}\left[\pi_{R}\right]$ will be called the Nielsen invariant modulo 2 (here ind ${ }_{2}(f, g: A)$ denotes index modulo 2 of the class $A$, that is, the parity of \#A if $f$ and $g$ are transverse).

The following two lemmas are due to Bo Ju Jiang.

LEMMA 3.4. ([10, (4.1)]) Suppose $g, g^{\prime} \in G$ and $g=g^{\prime} \bmod K$. Then for any $k \in K$ the elements $k^{g}$ and $k^{g^{\prime}}$ are conjugate in $K$. In particular $B^{g}={ }_{2} B^{g^{\prime}}$ where $=_{2}$ denotes equality in $K_{a b}(K$ abelianized).

LEMMA 3.5. ([10, (4.2)]) Suppose $h \in H$. Then $B^{\rho_{1 i} h}={ }_{2} B^{ \pm h \rho_{2 i}^{-1}}$ with minus sign if and only if the band is twisted.

PROOF. Follow [10, (4.2)], recalling only that our exponential convention $B^{v}$ is not consistent with that of Jiang. 
COROLLARY 3.6. (Compare [10, (4.3)]) If $k=k\left(\rho_{1 s}\right), h=h\left(\rho_{2 s}\right)$ are words then $B^{k h}={ }_{2} B^{ \pm h k^{\prime-1}}$ where $k^{\prime}=k\left(\rho_{2 s}\right)$ and where the sign is minus if and only if the path $k\left(r_{1 s}\right)$ reverses orientation of $N$.

The isomorphism $\beta_{S}: \hat{Z}[\pi] \rightarrow K$ induces the isomorphism $\beta: Z[\pi] \rightarrow K_{a b}$ which does not depend on the Schreier transversal ([10, Lemma (4.1)]). We denote by $\lambda: K_{a b} \rightarrow Z[\pi]$ its inverse.

THEOREM 3.7. Let $M$ and $N$ be oriented. The composition

$$
\lambda_{R}: K \rightarrow K_{a b} \rightarrow Z[\pi] \rightarrow Z\left[\pi_{R}\right]
$$

sends $R\left(u_{j} \sigma_{j}\right)$ (from Lemma 2.1) into the Nielsen invariant (here we identify $\pi=H / K$ with $\pi_{\mathrm{l}}\left(N, g x_{0}\right)$ by $\left.p_{2 \#}\right)$.

PROOF. We may assume that $(f, g)$ is of form as in Lemma 1.2. Recall our convention: two paths in $N$ with ends in $\bar{U}$ will be called homotopic if and only if they represent homotopic loops in the quotient space $N / \bar{U}$ ( $\bar{U}$ contracted to a point). Now it follows from the proof of Lemma 1.2 that if $\bar{a}_{j}$ is a path in $D$ from $x_{0}$ to $x_{j}$ then (following the above convention) $\left\langle f \bar{a}_{j}\right\rangle=p_{1 *}\left\langle v_{j}\right\rangle,\left\langle g \bar{a}_{j}\right\rangle=p_{2^{*}}\left\langle v_{j}\right\rangle$. Thus

$$
I\left(x_{j}\right)=\left\langle\left(g \bar{a}_{j}\right)\left(f \bar{a}_{j}^{-1}\right)\right\rangle_{R}=\left\langle\left(p_{2} v_{j}\right)\left(p_{1} v_{j}^{-1}\right)\right\rangle_{R} \in \pi_{R} .
$$

Let us denote

$$
\begin{aligned}
& \left\langle p_{1} v_{j}\right\rangle=F_{j}\left(r_{1 s}\right) \in \pi_{1}\left(N, f x_{0}\right) \\
& \left\langle p_{2} v_{j}\right\rangle=G_{j}\left(r_{2 s}\right) \in \pi_{1}\left(N, g x_{0}\right)
\end{aligned}
$$

and let $w_{j}=F_{j}\left(\rho_{1 s}\right) G_{j}\left(\rho_{2 s}\right), z_{j}=G_{j}\left(\rho_{2 s}\right) F_{j}\left(\rho_{2 s}\right)^{-1}$. The element corresponding to $I\left(x_{j}\right)$ in $\pi_{R}=(H / K)_{R}$ is determined by $z_{j}$ since $p_{2 \sharp} z_{j}=\left\langle\left(p_{2} v_{j}\right)\left(p_{1} v_{j}^{-1}\right)\right\rangle=$ $\left\langle g\left(\bar{a}_{j}\right) f\left(\bar{a}_{j}\right)^{-1}\right\rangle$. We notice that $v_{j}=w_{j} \bmod K$ (since their projections $p_{1 \#}, p_{2 \# \text { are }}$ equal) hence by Lemma $3.4 B^{v_{j}}={ }_{2} B^{w_{j}}$. On the other hand, by Corollary 3.6, $B^{w_{j}}={ }_{2} B^{z_{j}}$. Thus $\lambda\left(B^{v_{j}}\right)=z_{j}$ and at last

$$
\begin{aligned}
\lambda_{R}\left(R\left(u_{j} \sigma_{j}\right)\right) & =\lambda_{R}\left(B^{i_{1} v_{1}+\ldots+i_{k} v_{k}}\right)=\sum_{j=1}^{k} \lambda_{R}\left(B^{i_{j} v_{j}}\right)=\sum_{j=1}^{k} i_{j}\left[\left\langle z_{j}\right\rangle\right]_{R} \\
& =\sum_{j=1}^{k} \operatorname{ind}\left(f, g: x_{j}\right) I\left(x_{j}\right)=\sum \operatorname{ind}(f, \dot{g}: A) I(A) \in Z\left[\pi_{R}\right]
\end{aligned}
$$

where the summation runs over all Nielsen classes. 
REMARK 3.8. When $M$ or $N$ is not orientable then we may follow the above proof using the index $\bmod 2$. Then the composition

$$
\lambda_{R}: K \rightarrow K_{a b} \stackrel{\lambda}{\rightarrow} Z[\pi] \rightarrow Z\left[\pi_{R}\right] \rightarrow Z_{2}\left[\pi_{R}\right]
$$

sends $R\left(u_{j} \sigma_{j}\right)$ into the Nielsen invariant modulo 2 .

\section{Sphere and projective plane}

As we have seen, two of the simplest surfaces - the sphere and projective plane - are excluded from the above theory and they need an individual aproach. We will show at first that any Nielsen class of maps into these spaces may be reduced to a single point and then that any inessential class may be removed. This will imply

THEOREM 4.0. Any pair of maps $f, g$ into $S^{2}$ or $R P^{2}$ is homotopic to a pair with $N(f, g)$ coincidence points.

(Here $N(f, g)$ denotes Nielsen number introduced in [3]).

We will focus on maps into $R P^{2}$. The case of the sphere is simpler and can be easily deduced.

DEFINITION 4.1. The pair of homotopies $F_{t}, G_{t}: M \rightarrow N$ will be called special if and only if $\Phi\left(F_{t}, G_{t}\right)$ does not depend on $t \in[0,1]$.

The proofs of the above two lemmas are modifications of the well known ones for fixed points.

Lemma 4.2. (Compare [7, 2.1]) Let $X, Y$ be compact ANRs and let $A \subset U \subset X$, with A-closed and $U$-open. Let the maps $f, g: X \rightarrow Y$ and special homotopies $F_{t}^{\prime}, G_{t}^{\prime}: A \rightarrow Y_{0}$ satisfy $F^{\prime}(a)=f a, G_{0}^{\prime}(a)=$ ga for $a \in A$. Then $F_{t}^{\prime}, G_{t}^{\prime}$ may be extended to the whole of $X$ giving special homotopies $F_{t}, G_{t}$ satisfying $F_{t}(x)=f(x)$, $G_{t}(x)=g(x)$ for $(x, t) \in X \times 0 \cup(X-U) \times I$.

LEMMA 4.3. (Compare [12,1.2,1.3]) Let $f, g: M \rightarrow N$ and let $\omega$ be an arc in $M$ joining two isolated coincidence points $x_{0}$ and y such that $\Phi(f, g) \cap \omega[0,1]=\left\{x_{0}, y\right\}$. Let $f, g$ be near on $\omega$. Then for any neighbourhood $U$ of $\omega[0,1] f, g$ is homotopic rel $M-U$ to a pair $f^{\prime}, g^{\prime}$ of maps which are near on $\omega$ and $\Phi\left(f^{\prime}, g^{\prime}\right)=\Phi(f, g)-x_{0}$.

LEMMA 4.4. Let the paths $\alpha, \beta:[0,1] \rightarrow R P^{2}$ satisfy $\alpha(t)=\beta(t)$ if and only if $t \in\{0,1\}$ and be homotopic. Then they are specially homotopic (hence $(\alpha, \beta)$ is specially homotopic to a near pair). 
PROOF. Consider the lifts satisfying $\tilde{\alpha}(0)=\tilde{\beta}(0)$

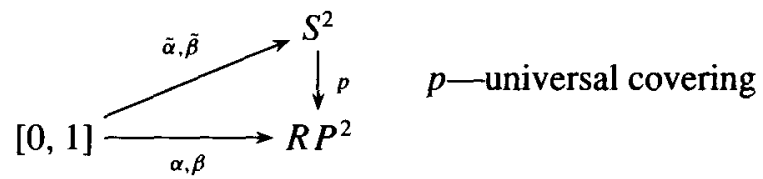

Since $\alpha$ and $\beta$ are homotopic, $\alpha(1)=\beta(1)$ and since $\alpha(t) \neq \beta(t)(0<t<1), \tilde{\alpha}(t)$ and $\tilde{\beta}(t)$ are never antipodal. Now $\tilde{\alpha}$ and $\tilde{\beta}$ are specially homotopic (by geodesics) hence so are $\alpha$ and $\beta$.

LEMMA 4.5. Let $f, g: M \rightarrow R P^{2}$ with $\Phi(f, g)$ finite. If the points $x, y \in \Phi(f, g)$ are Nielsen related then there is an arc between them establishing this relation.

PROOF. This follows from the fact that any path $\omega$ on a surface satisfying $\omega(0) \neq$ $\omega(1)$ is fixed end point homotopic to an arc [7, 3.3].

Let $f, g: M \rightarrow R P^{2}$. We may assume that $\Phi(f, g)$ is finite. Let $x, y \in \Phi(f, g)$ be Nielsen related. By Lemma 4.5 we can find an arc joining them, establishing the Nielsen relation and moreover avoiding other coincidence points. Now Lemma 4.4 and Lemma 4.3 reduce these two points to one. In this way we obtain one point in each Nielsen class.

It remains to show that any inessential class $A$ of the pair $f, g: M \rightarrow R P^{2}$ may be removed. By the above we may assume that $A$ is a single point. In the oriented case ind $(f, g: A)=0$ and this coincidence class disappears after a local deformation. But the following example shows that in the non-orientable case an inessential one point class may be of non-zero local index.

EXAMPLE. Let $D^{2}=\{z \in C:|z| \leq 1\}$ and let us present $R P^{2}=D^{2} / \cong(z \cong$ $-z$ for $|z|=1$ ). Let $f, g$ be self maps of $R P^{2}$ given by $f[z]=[0]$ (constant map) and $g[z]=\left[z^{2}\right]$. Then $g_{t}[z]=\left[t z^{2}\right]$ shows that $g$ is also homotopic to a constant map; hence $N(f, g)=0$. But on the other hand $\Phi(f, g)=\{[0]\}$, and the local index of this point is \pm 2 .

We will see that the situation of the above example occurs for defective Nielsen classes only [6] and we will show how to remove such classes.

DEFINITION 4.6. We will say that $\alpha \in \pi_{1} M$ satisfies the condition $Z$ if and only if $f_{\#} \alpha=g_{\#} \alpha$ and exactly one $\alpha$ or $f_{\#} \alpha$ preserves the orientation.

Recall that a Nielsen class is defective if and only if there is a loop based at this class satisfying $(Z)[6]$. 
Let $A=\left\{x_{0}\right\}$ be a Nielsen class of $f, g: M \rightarrow R P^{2}$. Let us fix local orientations at the points $x_{0} \in M, f x_{0}=g x_{0} \in R P^{2}$ and let $n \in Z$ be the local coincidence index with the respect to these orientations (we may assume $n \geq 0$ ). By a small deformation we may replace $x_{0}$ by $\mathrm{n}$ coincidence points $x_{1}, \ldots, x_{n}$ all of the index +1 . Since $A$ is inessential, the point $x_{1}$ reduces some $x_{i}$ and since they lie in the same euclidean neighbourhood mapped into another euclidean neighbourhood, $x_{1}$ reduces itself. Thus the class $A$ is defective and its local index is even.

LEMMA 4.7. Let $f, g: M \rightarrow N$ and let $A=\{a\}$ be an inessential Nielsenclass. Let $\omega$ be a closed arc based at a satisfying $(Z), w(t) \notin \Phi(f, g)$ for $0<t<1$ and $f \omega, g \omega$ are specially homotopic to a near pair. Then for any open subset $U$ containing $\omega[0,1]$, $f, g$ is homotopic rel $(M-U)$ to a pair $f^{\prime}, g^{\prime}$ satisfying $\Phi\left(f^{\prime}, g^{\prime}\right)=\Phi(f, g)-A$.

PROOF. By Lemma 4.3 the special homotopy on $\omega$ may be extended onto $M$ so we may assume that $f \omega, g \omega$ are near. Since $A$ is inessential its local index must be even, say $2 k$. We may split $a$ into two coincidence points $a$ and $a^{\prime}$ both of local index $k$. Then we translate $a$ (as in Lemma 4.3) along the path $\omega$ and we get the coincidence point $a$ again but now of local index - $k$ (since exactly one of $\omega$ or $f \omega$ preserves the orientation). Now the points $a$ and $a^{\prime}$ cancel one another.

It remains to prove that if $A=\{a\}$ is a defective inessential Nielsen class of $f, g: M \rightarrow R P^{2}$ then there exists a closed arc based at a satisfying the hypothesis of 4.6. This follows from

LEMMA 4.8. For any defective Nielsen class of $f, g: M \rightarrow R P^{2}$ there is an element satisfying condition $(Z)$ being the composition of at most two generators. In particular, it may be represented by a closed arc in $M$.

PROOF. Suppose otherwise. Let $\alpha=\alpha_{1} \ldots \alpha_{k}$ satisfy (Z) $\left(\alpha_{1}, \ldots, \alpha_{k}\right.$ being generators). Since (Z) does not hold for generators, $f_{\#} \alpha_{i}=g_{\#} \alpha_{i}$ implies both $\alpha_{i}$ and $f_{\#} \alpha_{i}$ simultaneously preserve or reverse the orientation. We may drop such $\alpha_{i}$ and assume $f_{\#} \alpha_{i} \neq g_{\#} \alpha_{i}$ for $i=1, \ldots, k$. Now $f_{\#}\left(\alpha_{1} a_{2}\right)=g_{\#}\left(\alpha_{1} a_{2}\right)$ and for the same reasons as above we may drop $\alpha_{1} a_{2}$. Following this procedure we obtain $k \leq 2$.

\section{References}

[1] R. Brooks, 'On removing coincidences of two maps when only one, rather than both, of them may be deformed by a homotopy', Pacific J. Math. 40 (1972), 45-52.

[2] R. F. Brown, The Lefschetz fixed point theorem (Scott, Foresman and Co., Glenview, 1971). 
[3] R. Dobreńko and J. Jezierski, 'The coincidence Nielsen number on non-orientable manifolds', Rocky Mountain J. Math. 23 (1993), 67-85.

[4] J. Jezierski, 'The Nielsen number product formula for coincidences', Fund. Math. 134 (1989), 183-212.

[5] — - 'The coincidence Nielsen number for maps into real projective spaces', Fund. Math. 140 (1992), 121-136.

[6] _ 'The semi-index product formula', Fund. Math. 140 (1992), 99-120.

[7] B. J. Jiang, 'On the Nielsen number of fixed points', Amer. J. Math. 102 (1980), 749-763.

[8] _ - 'Lectures on the Nielsen fixed point theory', Contemp. Math. 14 (Amer. Math. Soc., Providence, 1983).

[9] —_, 'Fixed points and braids', Invent. Math. 75 (1985), 69-74.

[10] _ - 'Surface maps and braid equations, I', Lecture Notes in Math. 1369 (Springer, NewYork, 1989) pp. 125-141.

[11] P. Olum, 'Obstructions to extensions and homotopies', Ann. of Math. (2) 52 (1950), 1-50.

[12] G. H. Shi, 'On the least number of fixed points and Nielsen numbers', Acta Math. Sinica 16 (1966), 223-232 (Chinese Math. Acta 8 (1966), 234-243).

\section{Department of Mathematics}

University of Agriculture

Nowoursynowska 166

02766 Warsaw

Poland 